\title{
STIL is upregulated in nasopharyngeal carcinoma tissues and promotes nasopharyngeal carcinoma proliferation, migration and invasion
}

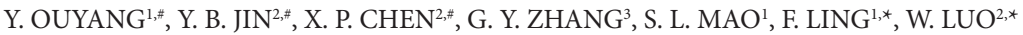 \\ ${ }^{1}$ School of Biology and Biological Engineering, South China University of Technology, Guangzhou 510006, China; ${ }^{2}$ Clinical Research Institute, \\ The First People's Hospital of Foshan, Sun Yat-sen University, Foshan 528000, Guangdong, China; ${ }^{3}$ Head and Neck Cancer Research, Depart- \\ ment of Otolaryngology, Head and Neck Surgery, Foshan Hospital, Sun Yat-sen University, Foshan 528000, Guangdong, China
}

*Correspondence: fling@scut.edu.cn; luowei_421@163.com

"Contributed equally to this work.

Received March 6, 2019 / Accepted May 22, 2019

\begin{abstract}
Nasopharyngeal carcinoma (NPC) is the most common primary malignancy that originates from the nasopharynx. Some regulatory networks involved in nasopharyngeal carcinoma have been reported, but the relevant genes have not been fully identified. We have used mRNA microarray to identify differential expression genes between NPC tissues and adjacent normal tissues. Then high-content shRNA screening was carried out to screen the genes that may control proliferation in nasopharyngeal carcinoma. Cell proliferation was monitored by MTT assays and Celigo image cytometry in vitro and by subcutaneous transplantation model in vivo. Flow cytometric analysis was carried out to detect the distribution of cell cycle stages and apoptosis. Transwell assay was performed to measure the migratory and invasive capacities of NPC cells. We identified 20 genes that potentially play an important role in the proliferation of nasopharyngeal carcinoma by mRNA microarray and functional analysis. The result of high-content shRNA screening indicated that STIL had the greatest effect on reducing the proliferation rate of NPC cells. The analysis of The Cancer Genome Atlas (TCGA) data showed that STIL was upregulated in several human cancer tissues, and higher STIL expression level was correlated with shorter survival time. STIL knockdown also inhibited NPC cell migration and invasion, promoted G1/S phase transition and apoptosis. Three genes including ITGA2, SMAD2, JAK1, associated with molecular mechanisms of cancer were influenced by downregulating STIL. Our study confirmed STIL as a key regulator that promotes the proliferation of NPC, providing insight into the molecular mechanisms of nasopharyngeal carcinoma and suggesting a novel therapeutic strategy.
\end{abstract}

Key words: STIL, nasopharyngeal carcinoma, cell proliferation, high-content shRNA screening, mRNA microarray

Nasopharyngeal carcinoma (NPC) is generally acknowledged as being one of the most common primary malignancies that originates from the nasopharynx, with the highest incidence in southeast Asia [1]. Annually, more than $70 \%$ of the 87,000 new cases of NPC are diagnosed as locoregionally advanced disease worldwide [2]. Radiotherapy combined with chemotherapy is the standard treatment for locoregionally advanced NPC patients [3, 4]. Currently, locoregional control of NPC has improved substantially and distant metastasis is known to be the main reason for treatment failure [5, 6]. The tumor-node-metastasis (TNM) staging system is the key determinant for treatment decisions. NPC patients with the same TNM stage receive similar treatments, but $30-40 \%$ of patients eventually develop distant metastasis after treatment [7]. Hence, the current anatomical-based staging system is not sufficient to predict the distant metastasis. The carcinogenesis of NPC is a complex process that involves the accumulation of genetic and epigenetic alterations [5, 8]. Identifying genes associated with NPC rapid growth and distant metastasis might lead to novel therapeutic strategies or more accurate prognostic prediction.

To screen for abnormally overexpressed genes in NPC, six pairs of matched nasopharyngeal carcinoma and adjacent normal tissues were subjected to a gene expression profile microarray analysis. High-content shRNA screening was carried out to verify the function of the identified candidate genes. We found that the centriolar replication factor STIL, may play a vital role in NPC proliferation.

The human STIL gene was initially identified in a common chromosomal rearrangement in T-cell acute lymphoblastic leukemia and named SCL/TAL1 Interrupting Locus (SIL/ STIL) $[9,10]$. STIL contains conserved regions and interacts 
with several proteins, such as PLK4, CDK1, SASS6 and CPAP $[11,12]$. Increasing evidence indicates that upregulation of STIL in differentiated tissues triggers centrosomal amplification and is associated with an increased metastatic potential in multiple cancers [13-15]. Thus, targeting STIL may be a promising anticancer strategy.

In this study, we demonstrate that STIL can promote NPC proliferation in vitro and in vivo and find some STIL-interaction genes, which were involved in molecular mechanisms of cancer. This result provides insight into the molecular mechanisms of NPC and suggests a novel therapeutic strategy.

\section{Materials and methods}

Ethical statement. This study was approved by the Human Research Ethics Committees at the Affiliated Foshan Hospital of Sun Yat-Sen University (Guangdong, China), and was carried out in accordance with the principles embodied in the Declaration of Helsinki. All animal treatments were carried out according to the US Public Health Service Policy on the Humane Care and Use of Laboratory Animals. All surgical procedures were conducted under sodium pentobarbital anesthesia and every effort was made to minimize animal suffering.

Cell culture. CNE-2Z cell lines were purchased from Genechem (Shanghai, China). The cell line was previously authenticated via STR typing (Suppl. file 1). CNE-2Z cells were grown in RPMI1640 medium (Gibco, USA) supplemented with $10 \%$ fetal bovine serum (Gibco, USA) at $37^{\circ} \mathrm{C}$ in a humidified atmosphere containing $5 \% \mathrm{CO}_{2}$.

Human tissue samples. All clinical samples in this study were obtained from the Affiliated Foshan Hospital of Sun Yat-Sen University. Tumor and paired adjacent normal tissue specimens were collected from 6 patients diagnosed with NPC. None of them had received chemoradiotherapy or surgery. All of the tissue specimens were obtained during endoscopic biopsy of the nasopharynx. The adjacent non-tumor tissues were taken at least $1 \mathrm{~cm}$ apart from the nasopharyngeal carcinoma tissue.

RNA microarrays. Total RNA was extracted from the tissue samples and cell line using TRIzol (Invitrogen, USA). RNA quantity and quality were measured by NanoDrop ND-1000 and RNA integrity was assessed by standard denaturing agarose gel electrophoresis. Sample labeling and array hybridization were performed according to the Agilent One-Color Microarray-Based Gene Expression Analysis protocol (Agilent Technology).

High-content screening and cell growth curve analysis. CNE-2Z cells were transfected with a gene knockdown (GENE-KD) or negative control (NC) lentivirus and seeded into 96-well plates. GFP expression was observed using a fluorescence microscope. The cells were collected for further experiments when they reached $80-90 \%$ confluency. A total of 2,000 cells per well were analyzed once a day using a Celigo System (Nexcelom). Cells were quantified by measuring the green fluorescence signal in each well. Data were collected for statistical analysis using 5-day cell proliferation curves. Image analysis software was used to count the cells on the scanned image. The number of cells at each time point was compared with the cell count on day 1 to obtain a cell proliferation ratio for each time point for each group, and the fold change in proliferation was used to produce a cell growth curve. The cell proliferation ratio was calculated as follows: fold change (NC vs GENE-KD group) = proliferation ratio on day 5 for the NC group/proliferation ratio on day 5 for the GENE-KD group.

Animals. Female 4-week-old BALB/c nude mice were purchased from LingChang Bioscience (Shanghai, China). All mice were maintained under specific pathogen-free conditions.

Subcutaneous transplantation of $\mathrm{CNE}-2 \mathrm{Z}$ cells in BALB/c nude mice. Female 4-week-old BALB/c nude mice were used in all experiments. A total of $4 \times 10^{6}$ transfected cells were subcutaneously injected into the right armpit of $\mathrm{BALB} / \mathrm{c}$ nude mice. The weight of each mouse and the tumor diameter were measured every workday from day 8 postinjection. All mice were killed 2 weeks after injection.

Statistical analyses. Results for continuous variables are presented as means \pm S.D. unless otherwise stated. Comparisons between two groups were analyzed using the independent sample t-tests, paired $t$ test or Mann-Whitney $U$ tests if appropriate. Two-sided $\mathrm{p}$-value of $<0.05$ was considered statistically significant. These analyses were performed using Graphpad Prism software (version 5.1) and SPSS (version 20.0).

\section{Results}

Identification of differentially expressed mRNAs in tumor tissues compared with adjacent normal tissues. To identify critical gene that contributes to NPC tumorigenesis, NPC tissues and paired adjacent normal tissues from 6 patients were subjected to mRNA microarray analysis. As shown in Figure 1A, the results identified 3,191 upregulated and 2,546 downregulated mRNAs in the NPC tissues. On the basis of a functional analysis and literature review, 20 genes were found to potentially play an important role in the proliferation of cancer (Suppl. file 2).

Identification of STIL as a gene that promotes NPC proliferation. All 20 candidate genes were then silenced in CNE-2Z cells to examine the potential effect on the proliferation of NPC cells in vitro (Figure 1B, Suppl. file 3). Knockdown of two candidate genes, STIL and KLHL42, in CNE-2Z cells reduced the cell proliferation rate to $>2$ fold than negative control (Figures 1B and C). STIL knockdown showed the greatest effect on reducing the proliferation rate and thus we focused on STIL in subsequent studies.

Upregulation of STIL in tumor is associated with poor patient prognosis. Interestingly, we analyzed the TCGA databases and found that STIL expression was also signifi- 
A

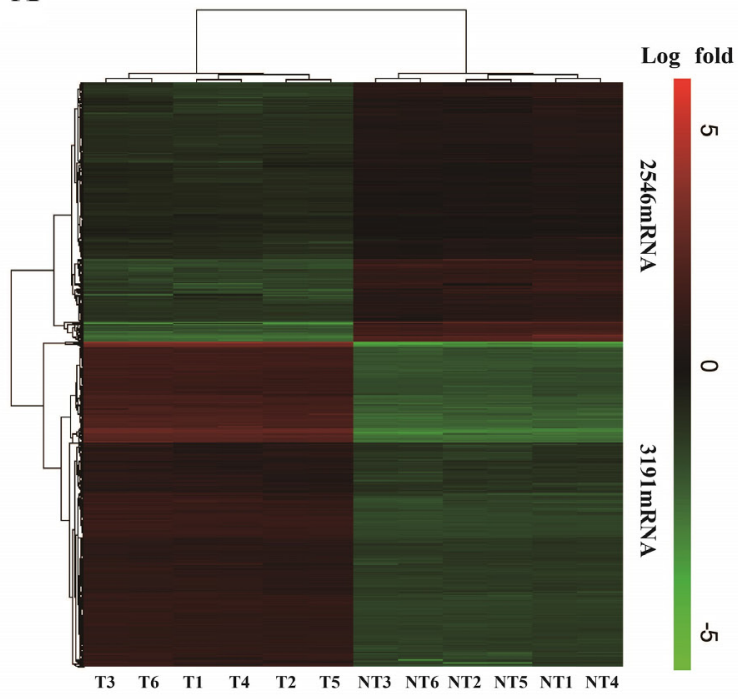

B

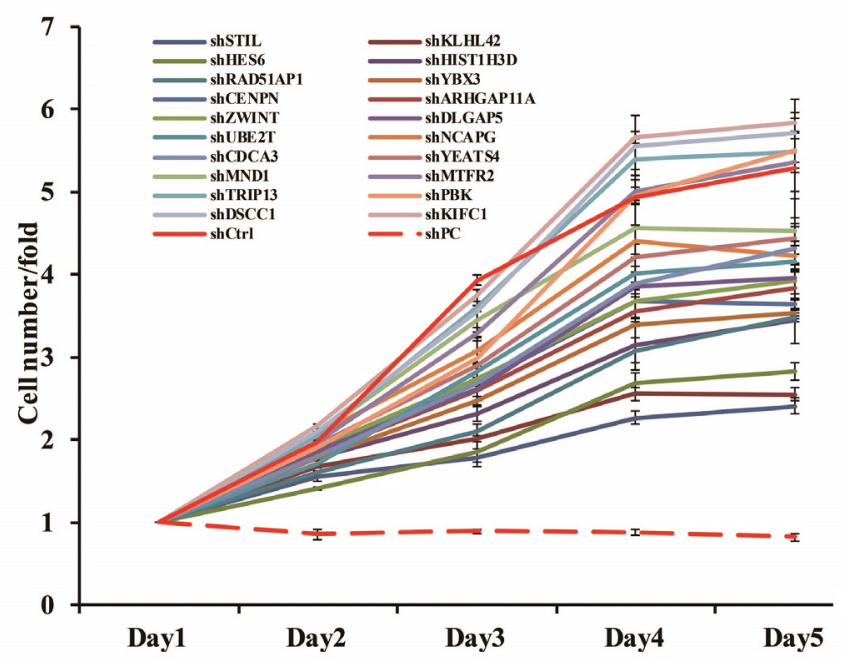

C
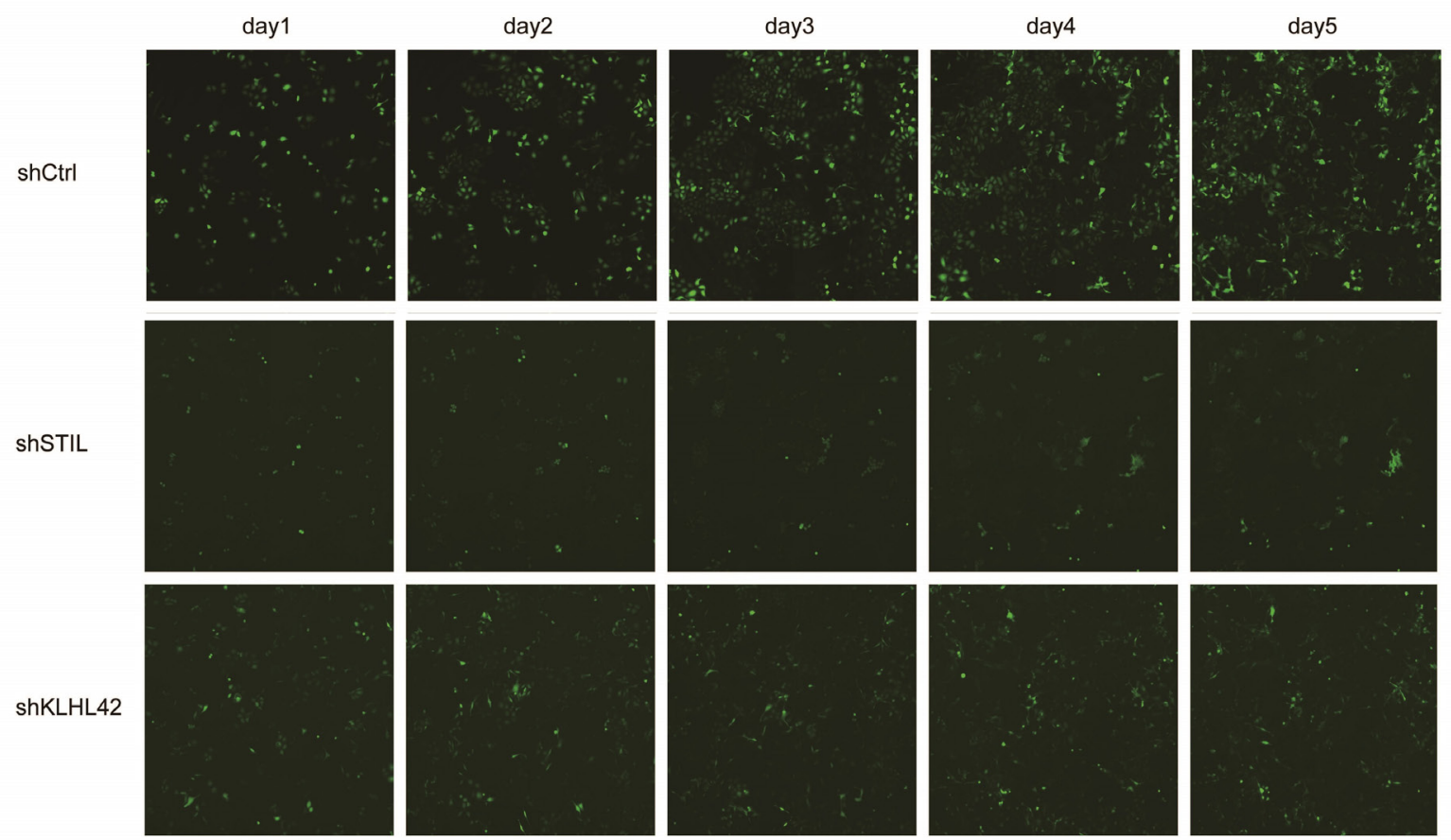

Figure 1. Agilent mRNA microarray and high-content shRNA screening identified STIL as a critical gene in promoting NPC proliferation. A) Heat map showing gene expression profiles. Each row represents a gene and each column represents a sample. Red indicates high expression, whereas green indicates low expression. T, tumor; NT, adjacent normal tissue. B) A total of 20 genes were selected for validation by high-content screening. NC: negative control shRNA, PC: positive control shRNA targeting $\beta$-actin. C) Representative fluorescence images of high-content shRNA screening for STIL and KLHL42.

cantly upregulated in glioblastoma multiforme, kidney renal clear cell carcinoma, head and neck squamous cell carcinoma, lung squamous cell carcinoma, liver hepatocellular carcinoma, breast invasive carcinoma and they are consistent with our results $[16,17]$ (Figures 2A-F). Moreover, higher expression of STIL is correlated with poor prognosis in kidney renal clear cell carcinoma and liver hepatocellular carcinoma [16], further supporting the oncogenic role of STIL in cancer (Figures $2 \mathrm{G}$ and $\mathrm{H}$ ).

Promotion of STIL in NPC cell proliferation, migration and invasion. To reveal the role of STIL in nasopharyngeal carcinoma progression, we designed a STIL shRNA 
A

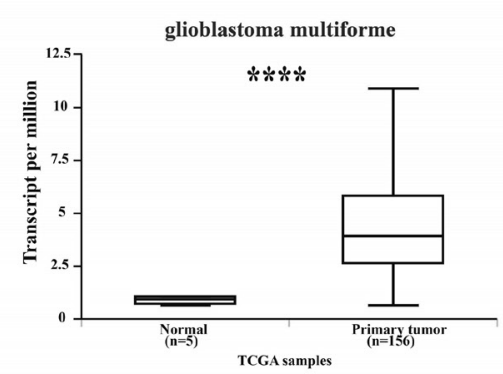

D

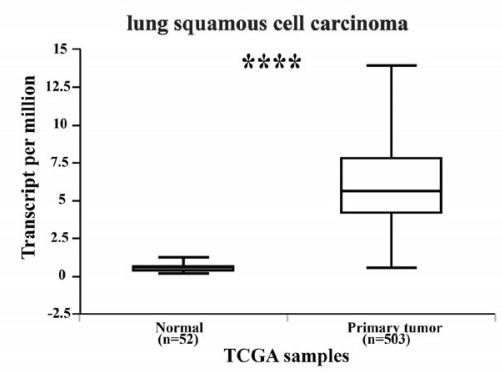

G

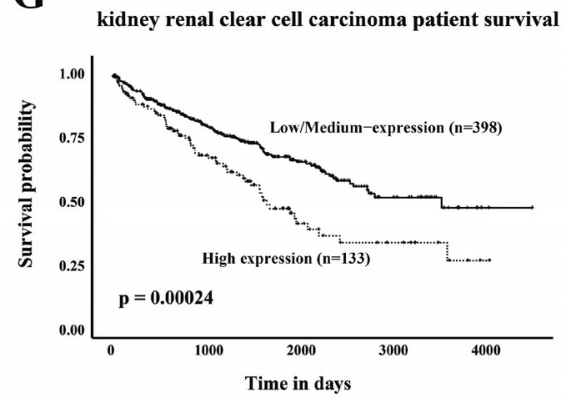

B

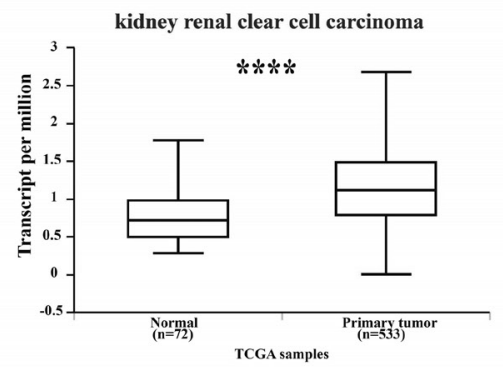

$\mathbf{E}$

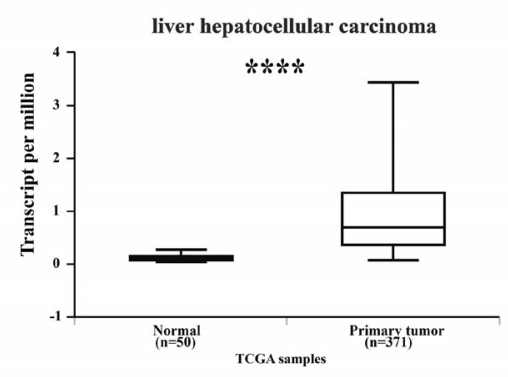

C

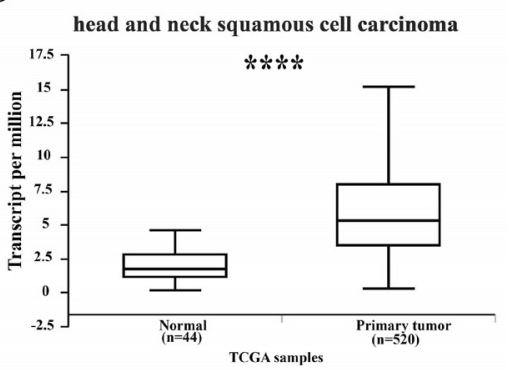

F

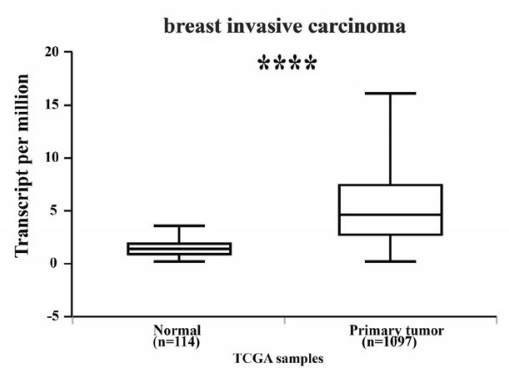

Figure 2. STIL is overexpressed in multiple types of human cancer. A-F) Data from TCGA databases were analyzed in different types of cancers as indicated. The Mann-Whitney U test was used to compare differences between the two groups. ${ }^{\star * * *} \mathbf{p}<0.0001$. G-H) TCGA data showed that STIL overexpression correlates with poor prognosis of kidney renal clear cell carcinoma and liver hepatocellular carcinoma.

with stable silencing efficiency by lentiviral transduction and confirmed downregulation of both STIL protein and mRNA in CNE-2Z cell line (Figures $3 \mathrm{~A}$ and B). Cell growth curve analysis using a fluorescence imaging system and MTT assay indicated that STIL knockdown suppressed NPC cell proliferation (Figure 3C and D). To further explore whether STIL plays a key role in NPC proliferation in vivo, a subcutaneous xenograft model of CNE-2Z cells in BALB/c nude mice was established. The STIL knockdown (STIL-KD) group showed a slower increase in tumor volume and weight compared with negative controls (Figures 3E-G). Furthermore, cell migration assay and cell invasion assay showed that STIL knockdown suppressed the migration and invasion of CNE- $2 \mathrm{Z}$ cells (Figure 4).
Promotion of G1/S phase transition and apoptosis and influence of the genes involving in cancer by STIL knockdown. We used flow cytometry to explore whether STIL promotes NPC proliferation through regulating the cell cycle and/or apoptosis. Flow cytometry analysis of the cell cycle in STIL-KD and NC CNE-2Z showed that the fraction of cells in $S$ phase increased, while the fraction of cells in G2/M phase decreased compared with NC group. Moreover, the apoptotic ratio increased significantly in STIL-KD group (Figures 5A and $C$ ). Independent experiments were performed to validate the results (Figure $5 \mathrm{~B}$ and $\mathrm{D}$ ).

To further elucidate the mechanisms underlying STIL promotion of NPC proliferation, mRNA microarray was performed in STIL-KD CNE-2Z cells and empty vector- 


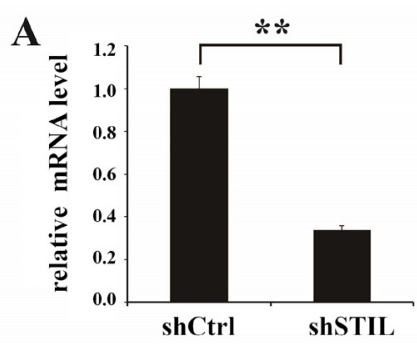

$\mathbf{E}$

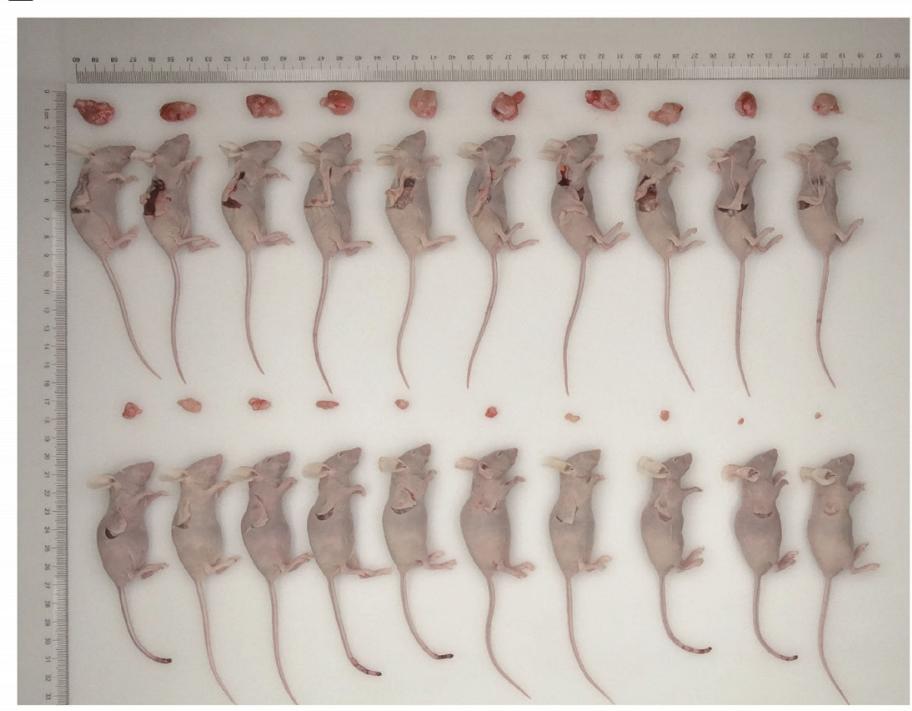

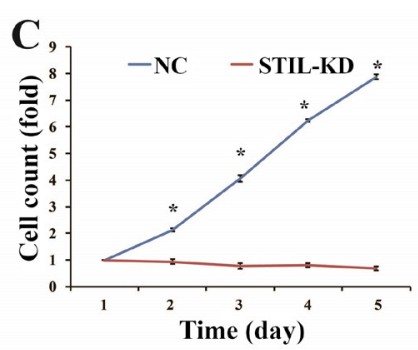
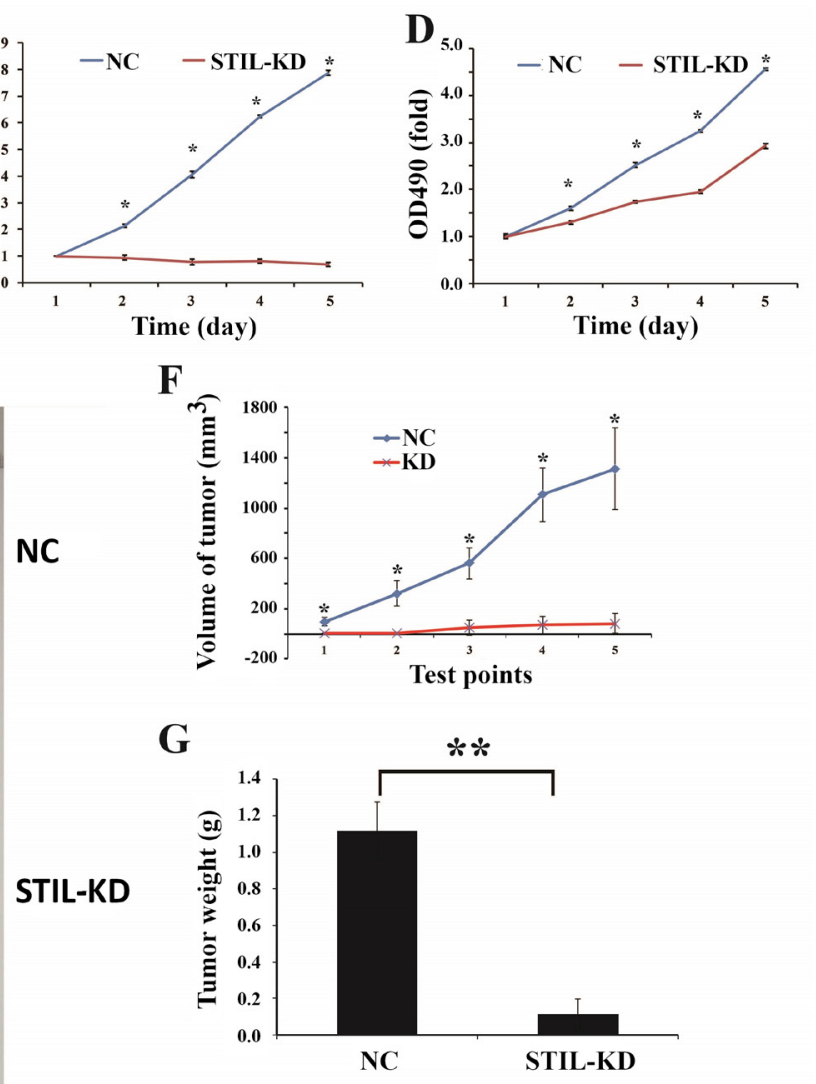

Figure 3. STIL knockdown affects NPC cell proliferation in vivo and in vitro. A and B) CNE-2Z cells were transfected with control (ctrl) shRNA, STIL1 shRNA. qRT-PCRs (A) and western blot (B) were performed to detect STIL levels. C) Cell growth curve analysis comparing STIL knockdown (STILKD) with negative control (NC) NPC cells. $n=3,{ }^{\star} p<0.05$. D) MTT assays comparing proliferation of STIL-KD and NC NPC cells. $n=3$, ${ }^{\star} p<0.05$. E) Images of the subcutaneous xenografts from the STIL-KD and NC groups. $n=10$. Tumor volume growth curves $(F)$ and tumor weight $(G)$ for subcutaneous xenografts. ${ }^{*} \mathrm{p}<0.05$. Tumor volume $=\pi / 6 \times$ long diameter $\times$ the short diameter $\times$ the short diameter. ${ }^{*} \mathrm{p}<0.05,{ }^{* *} \mathrm{p}<0.01$.

transduced cells. We found 325 upregulated and 457 downregulated genes with significant difference (|Fold Change $\mid>1.5$ and FDR $<0.05)$. Network analysis using data obtained from mRNA microarrays predicted some STILassociated genes (Figures 6A). Western blot and q-PCR analysis showed that ITGA2, ITGAV, SMAD2, JAK1 were downregulated significantly in STIL-KD cells (Figures 6B and C). Most of them (3/4, ITGA2, SMAD2, JAK1) are the key genes involved in molecular mechanisms of cancer, an ingenuity canonical pathway [18].

\section{Discussion}

The centrosome is a cytoplasmic organelle built around microtubule-based core components called centrioles. Centrosomes are essential for chromosomal stability, and abnormalities of their number, or structure affect cell division [12]. Many kinds of cancers are associated with centrosome dysfunction [13-15]. STIL gene encodes a regulatory protein necessary for centriole biogenesis and plays the central role in maintaining centrosome integrity in highly proliferating cells [11,13-15]. Overexpression of STIL, which results in supernumerary centrosomes, could lead to cancer because of chromosomal instability [19]. Although STIL has been found upregulated in several cancers, such as lung cancer, colon carcinoma, ovarian cancers and so on, the role of STIL in regulating the proliferation of nasopharyngeal carcinoma cells has not yet been elucidated.

STIL promoting SHH signaling could be another pathway to account for its association with cancer [11]. Sonic hedgehog $(\mathrm{SHH})$ is a morphogen involved in proliferation and survival of neural stem cells and STIL participates in the control of SHH signaling [20]. STIL can bind to suppressor-of-fused homolog (SUFU) and SUFU is a negative regulator of SHH [21]. In PC12 cells, a cell line derived from a pheochromocytoma of the rat adrenal medulla, downregulating STIL would decrease the SHH signaling and cell proliferation [22]. Similar results have been evidenced in other cancer cell lines, such as H1299, Hela, PC3 and LS174T cells [23]. These further suggested that STIL plays a role in cell proliferation through the SHH pathway [24]. 
A
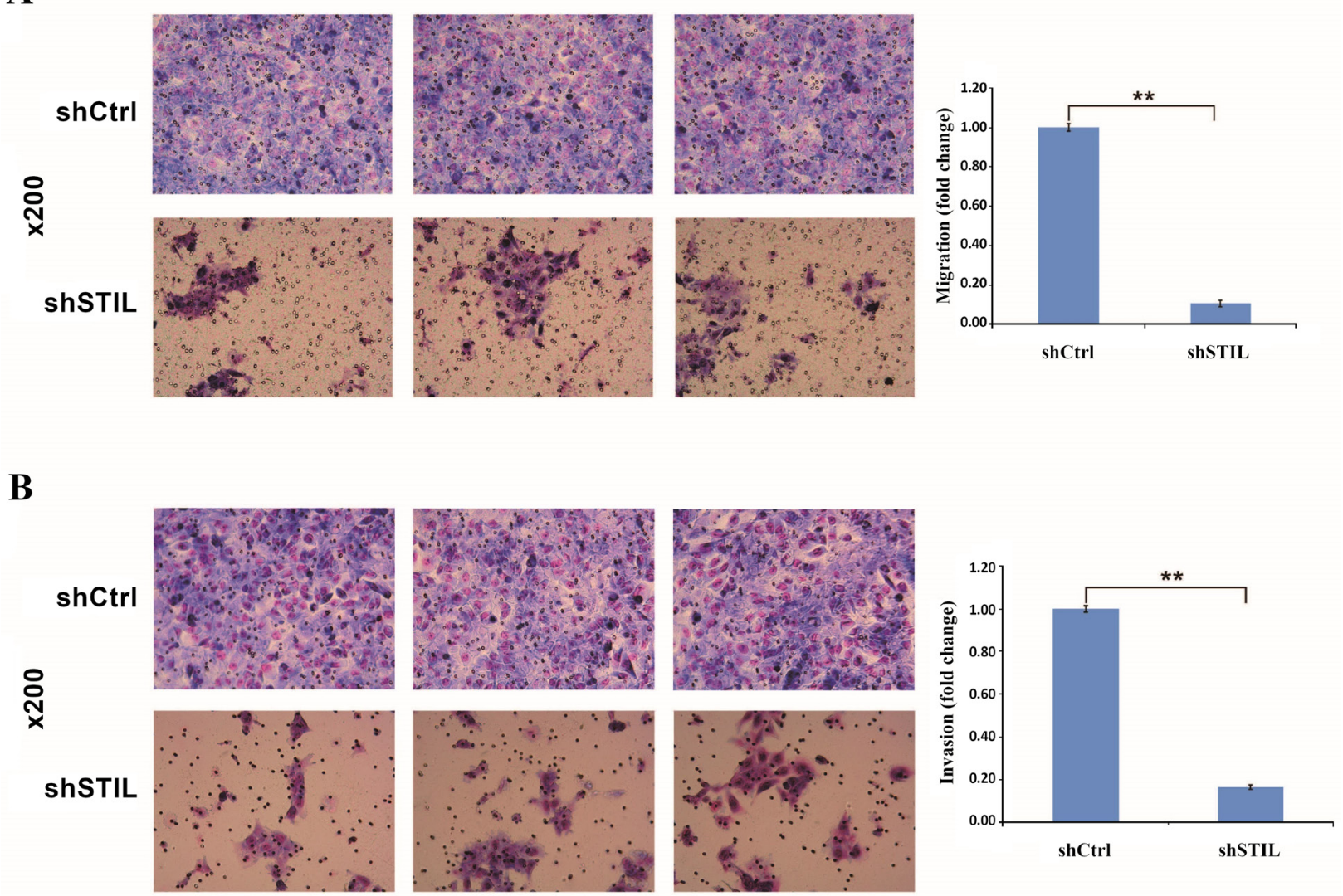

Figure 4. Transwell migration assay (A) and invasion assay (B) showed that STIL knockdown suppressed NPC cell migration and invasion. ${ }^{\star *}$ p $<0.01$.

A

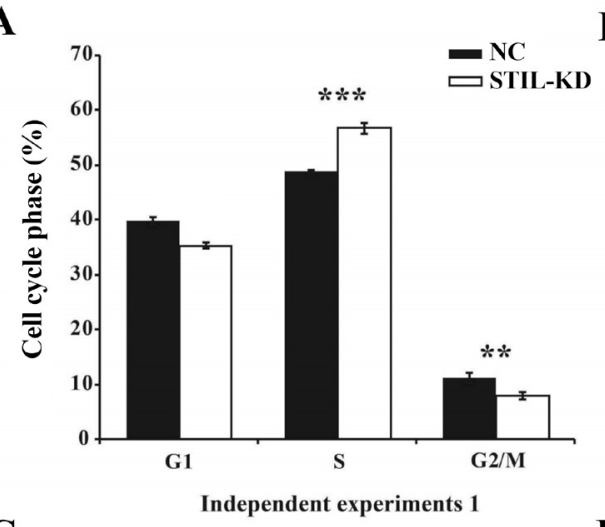

B

C

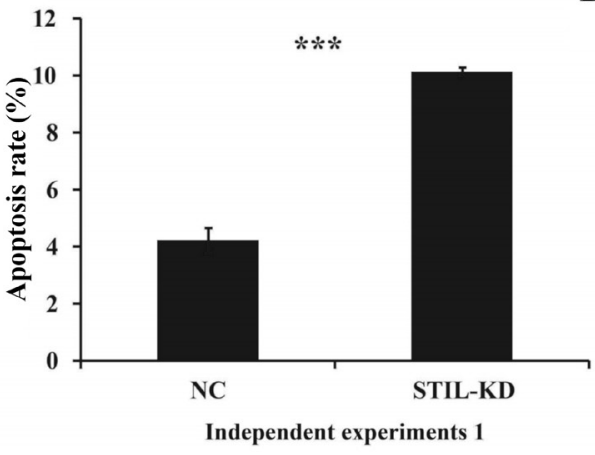

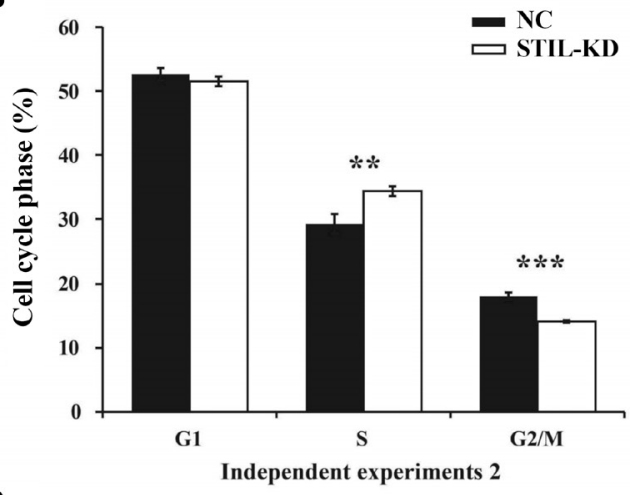

D

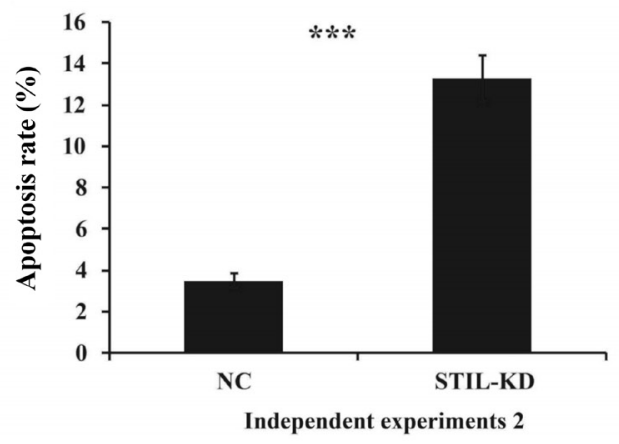

Figure 5. STIL knockdown promotes G1/S phase transition and apoptosis. $A$ and $B$ ) Flow cytometry analysis of the cell cycle in STIL knockdown (STIL-KD) and negative control (NC) CNE-2Z cells. C and D) Flow cytometry analysis of apoptosis in STIL-KD and NC CNE-2Z cells. $\mathrm{n}=3 .{ }^{\star *} \mathrm{p}<0.01,{ }^{* * *} \mathrm{p}<0.001$. 


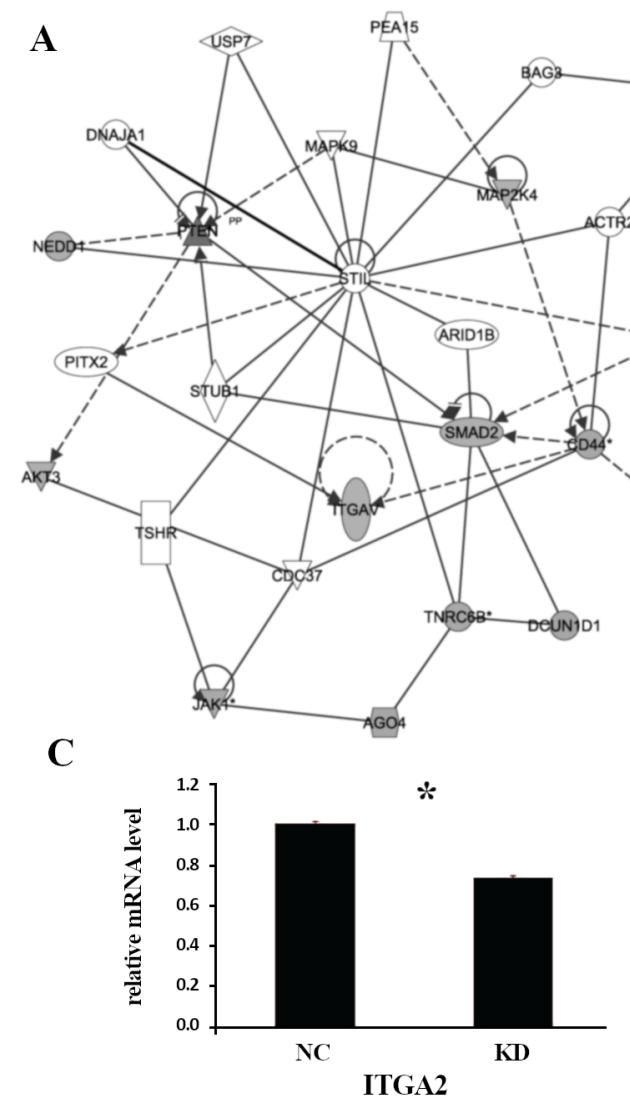

B
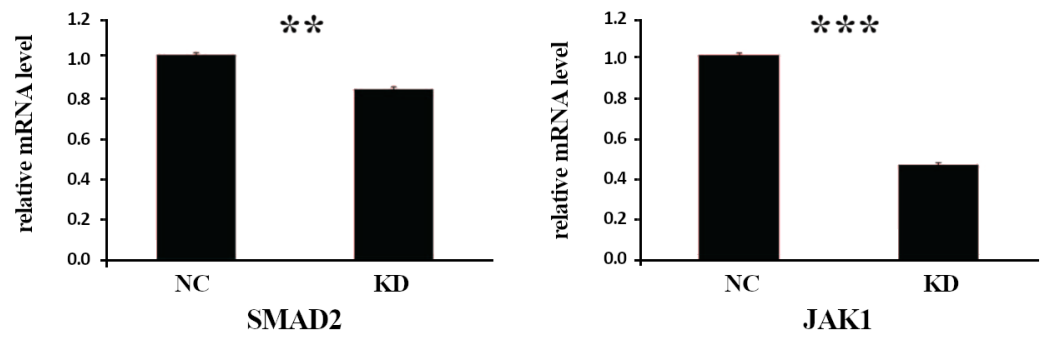

Figure 6. STIL knockdown influence the genes that participate in molecular mechanisms of cancer. A) Network analysis of STIL-associated genes using mRNA microarrays data obtained from STIL knockdown and empty vector-transduced CNE-2Z cells. B-C) Protein and relative mRNA expression levels (in relation to GAPDH) of STIL-associated genes were analyzed by western blotting (B) and qRT-PCRs (C) in STIL knockdown (KD) and negative control (NC) CNE-2Z cells. ${ }^{\star} \mathrm{p}<0.05,{ }^{\star *} \mathrm{p}<0.01,{ }^{* * *} \mathrm{p}<0.001$.

participate in centromeric replication and number regulation. Among them, CPAP/HsSAS-4 is required for centromeric replication; STIL/SIL binds to SAS- 6 to participate in the assembly of the original centrosome. Mutations in these proteins can cause centrosome abnormalities in varying degrees. In addition, the stability of STIL expression levels in the cytoplasm is affected by STILT (STIL truncating mutations). More importantly, STILT can also be clustered around and separated from the centrosome like STIL. The two genes play a consistent role in promoting centrosome synthesis, indicating that the true role of STILT is to affect centrosome amplification.
This study reports that STIL promotes the proliferation of NPC cells and STIL knockdown influences the NPC cell cycle and apoptosis. We also found that the expressions of 3 genes (ITGA2, SMAD2 and JAK1), which were associated with molecular mechanisms of cancer, were influenced by downregulating STIL. Based on the results of our research, we suggest that STIL may promote the migration and invasion of nasopharyngeal carcinoma through the mechanistic association with the three abovementioned genes. This study did not investigate the role of STIL in regulating centromere function during the metastasis in nasopharyngeal carcinoma, so it cannot be ruled out whether this metastatic activity responds 
to STIL's role in regulating centrosome biogenesis. To confirm further the role of STIL in nasopharyngeal carcinoma, we will next explore the oncogenic mechanism of STIL and the aforementioned three genes, and further discuss whether the role of STIL in regulating centrosome biogenesis will affect the metastasis activity of nasopharyngeal cancer cells.

In conclusion, our study provides evidence that supports STIL as a key regulator that promotes the proliferation of NPC. The result provides insight into the molecular mechanisms of nasopharyngeal carcinoma and suggests a novel therapeutic strategy.

Supplementary information is available in the online version of the paper.

Acknowledgements: This study was funded by Guangdong Province Natural Science Funds for Distinguished Young Scholar (2016A030306050) \& "Guangdong Te Zhi program" youth science and technology talent of project (2015TQ01R462) \& Science and technology innovation platform in Foshan city (2015AG10002) \& The Fundamental Research Funds for the Central Universities (x2swD2172910) \& Grants from National Natural Science Foundation of China (81402255). We would like to give our sincere gratitude to the reviewers for their constructive comments.

\section{References}

[1] TORRE LA, BRAY F, SIEGEL RL, FERLAY J, LORTETTIEULENT J et al. Global cancer statistics, 2012. CA Cancer J Clin 2015; 65: 87-108. https://doi.org/10.3322/ caac. 21262

[2] PAN JJ, NG WT, ZONG JF, LEE SW, CHOI HC et al. Prognostic nomogram for refining the prognostication of the proposed 8th edition of the AJCC/UICC staging system for nasopharyngeal cancer in the era of intensity-modulated radiotherapy. Cancer 2016; 122: 3307-3315. https://doi. org/10.1002/cncr.30198

[3] WIERZBICKA M, NAPIERALA J. Updated National Comprehensive Cancer Network guidelines for treatment of head and neck cancers 2010-2017. Otolaryngol Pol 2017; 71: 1-6. https://doi.org/10.5604/01.3001.0010.7193

[4] Zhang L, Chen Q Y, Liu H, Tang L Q,Mai H Q. Emerging treatment options for nasopharyngeal carcinoma. Drug Des Devel Ther 2013; 7: 37-52.

[5] TANG XR, LI YQ, LIANG SB, JIANG W, LIU F et al. Development and validation of a gene expression-based signature to predict distant metastasis in locoregionally advanced nasopharyngeal carcinoma: a retrospective, multicentre, cohort study. Lancet Oncol 2018; 19: 382-393. https://doi. org/10.1016/S1470-2045(18)30080-9

[6] LAI SZ, LI WF, CHEN L, LUO W, CHEN YY et al. How does intensity-modulated radiotherapy versus conventional two-dimensional radiotherapy influence the treatment results in nasopharyngeal carcinoma patients? Int J Radiat Oncol Biol Phys 2011; 80: 661-668. https://doi.org/10.1016/j. ijrobp.2010.03.024
[7] HUI EP, LEUNG SF, AU JS, ZEE B, TUNG S et al. Lung metastasis alone in nasopharyngeal carcinoma: a relatively favorable prognostic group. A study by the Hong Kong Nasopharyngeal Carcinoma Study Group. Cancer 2004; 101: 300-306. https://doi.org/10.1002/cncr.20358

[8] LO KW, HUANG DP. Genetic and epigenetic changes in nasopharyngeal carcinoma. Semin Cancer Biol 2002; 12: 451-462. https://doi.org/10.1016/S1044579X02000883

[9] APLAN PD, LOMBARDI DP, REAMAN GH, SATHER $\mathrm{HN}, \mathrm{HAMMOND}$ GD et al. Involvement of the putative hematopoietic transcription factor SCL in T-cell acute lymphoblastic leukemia. Blood 1992; 79: 1327-1333.

[10] D'ANGIO M, VALSECCHI MG, TESTI AM, CONTER V, NUNES V et al. Clinical features and outcome of SIL/TAL1positive T-cell acute lymphoblastic leukemia in children and adolescents: a 10-year experience of the AIEOP group. Haematologica 2015; 100: e10-13. https://doi.org/10.3324/haematol.2014.112151

[11] PATWARDHAN D, MANI S, PASSEMARD S, GRESSENS P, EL GHOUZZI V. STIL balancing primary microcephaly and cancer. Cell Death Dis 2018; 9: 65. https://doi. org/10.1038/s41419-017-0101-9

[12] PIHAN GA. Centrosome dysfunction contributes to chromosome instability, chromoanagenesis, and genome reprograming in cancer. Front Oncol 2013; 3: 277. https://doi. org/10.3389/fonc.2013.00277

[13] EREZ A, PERELMAN M, HEWITT S M, COJACARU G, GOLDBERG I et al. Sil overexpression in lung cancer characterizes tumors with increased mitotic activity. Oncogene 2004; 23: 5371-5377. https://doi.org/10.1038/ sj.onc. 1207685

[14] RABINOWICZ N, MANGALA LS, BROWN KR, CHECARODRIGUEZ C, CASTIEL A et al. Targeting the centriolar replication factor STIL synergizes with DNA damaging agents for treatment of ovarian cancer. Oncotarget 2017; 8: 27380-27392. https://doi.org/10.18632/oncotarget.16068

[15] RAMASWAMY S, ROSS KN, LANDER ES, GOLUB TR. A molecular signature of metastasis in primary solid tumors. Nat Genet 2003; 33: 49-54. https://doi.org/10.1038/ng1060

[16] CHANDRASHEKAR DS, BASHEL B, BALASUBRAMANYA SAH, CREIGHTON CJ, PONCE-RODRIGUEZ I et al. UALCAN: A Portal for Facilitating Tumor Subgroup Gene Expression and Survival Analyses. Neoplasia 2017; 19: 649658. https://doi.org/10.1016/j.neo.2017.05.002

[17] AKBANI R, NG PK, WERNER HM, SHAHMORADGOLI $\mathrm{M}$, ZHANG $\mathrm{F}$ et al. A pan-cancer proteomic perspective on The Cancer Genome Atlas. Nat Commun 2014; 5: 3887. https://doi.org/10.1038/ncomms4887

[18] AHMADI Y, GHORBANIHAGHJO A, ARGANI H. The balance between induction and inhibition of mevalonate pathway regulates cancer suppression by statins: A review of molecular mechanisms. Chem Biol Interact 2017; 273: 273-285. https://doi.org/10.1016/j.cbi.2017.06.026

[19] ARQUINT C, NIGG EA. The PLK4-STIL-SAS-6 module at the core of centriole duplication. Biochem Soc Trans 2016; 44: 1253-1263. https://doi.org/10.1042/BST20160116 
[20] CHOUDHRY Z, RIKANI AA, CHOUDHRY AM, TARIQ S, ZAKARIA F et al. Sonic hedgehog signalling pathway: a complex network. Ann Neurosci 2014; 21: 28-31. https://doi. org/10.5214/ans.0972.7531.210109

[21] KASAI K, INAGUMA S, YONEYAMA A, YOSHIKAWA K, IKEDA H. SCL/TAL1 interrupting locus derepresses GLI1 from the negative control of suppressor-of-fused in pancreatic cancer cell. Cancer Res 2008; 68: 7723-7729. https://doi. org/10.1158/0008-5472.CAN-07-6661

[22] SUN L, CARR A L, LI P, LEE J, MCGREGOR M et al. Characterization of the human oncogene SCL/TAL1 interrupting locus (Stil) mediated Sonic hedgehog (Shh) signaling transduction in proliferating mammalian dopaminergic neurons. Biochem Biophys Res Commun 2014; 449: 444-448. https:// doi.org/10.1016/j.bbrc.2014.05.048

[23] EREZ A, CASTIEL A, TRAKHTENBROT L, PERELMAN M, ROSENTHAL E et al. The SIL gene is essential for mitotic entry and survival of cancer cells. Cancer Res 2007; 67: 40224027. https://doi.org/10.1158/0008-5472.CAN-07-0064
[24] SUN L, LI P, CARR A L, GORSUCH R, YARKA C et al. Transcription of the SCL/TAL1 interrupting Locus (Stil) is required for cell proliferation in adult Zebrafish Retinas. J Biol Chem 2014; 289: 6934-6940. https://doi.org/10.1074/ jbc.M113.506295

[25] LEVINE M S, BAKKER B, BOECKX B, MOYETT J, LU $J$ et al. Centrosome Amplification Is Sufficient to Promote Spontaneous Tumorigenesis in Mammals. Dev Cell 2017; 40: 313-322 e315. https://doi.org/10.1016/j.devcel.2016.12.022

[26] MOYER TC, CLUTARIO KM, LAMBRUS BG, DAGGUBATI V, HOLLAND AJ. Binding of STIL to Plk4 activates kinase activity to promote centriole assembly. J Cell Biol 2015; 209: 863-878. https://doi.org/10.1083/jcb.201502088

[27] KAZAZIAN K, GO C, WU H, BRASHAVITSKAYA O, XU R et al. Plk4 Promotes Cancer Invasion and Metastasis through Arp2/3 Complex Regulation of the Actin Cytoskeleton. Cancer Res 2017; 77: 434-447. https://doi.org/10.1158/00085472.CAN-16-2060 


\title{
Cell Line CNE-2Z STR Profile Report
}

\author{
Cell Line Designation: $\quad$ CNE-2Z \\ Date Sample Received: Dec $01^{\text {th }}, 2017$ \\ Report Date: $\quad$ Dec $05^{\text {th }}, 2017$
}

Methodology: $\quad$ Twenty short tandem repeat (STR) loci plus the gender determining locus,Amelogenin, were amplified using the commercially available PowerPlex® 21 System from Promega Corporation. The amplified products were processed using the Applied Biosystems 3730xl DNA Analyzer. Data were analyzed using GeneMapper 5.0 software (Applied Biosystems). Appropriate positive and negative controls were run and confinhed for each sample sulmitted

Data Interpretation: Cell lines were authenticated using Short Tandem Repeat (STR) analysis as described in 2012 in ANSI Standard (ASN-0002) by the ATCC Standards Develdpment Organization (SDO) and in Capes-Davis et al. Match criteria for human cell line authentication: Where do we draw the line? Int J Cancer.20/3,132(11):2510-9.
GENECHEM performs STR Profiling following ISO 9001:2008 and ISO/IEC 17025:2005 quality
standards. There are no warranties with respect to the services or results supplied, express or implied, including, without limitation, any implied warranty of merchantability or fitness for a particular purpose. GENECHEM is not liable for any damages or injuries resulting from receipt and/or improper, inappropriate, negligent or other wrongful use of the test results supplied, and/or from misidentification, misrepresentation, or lack of accuracy of those results. Your exclusive remedy against GENECHEM and those supplying materials used in the services for any losses or damage of any kind whatsoever, whether in contract, tort, or otherwise, shall be, at GENECHEM 's option, refund of the fee paid for such service or repeat of the service. 
TEST RESULTS:

\begin{tabular}{|c|c|c|}
\hline \multicolumn{2}{|c|}{ Test Results for Submitted Sample } & \multirow{2}{*}{$\begin{array}{c}\text { Reference Database Profile } \\
\text { Database Profile: CNE-2Z }\end{array}$} \\
\hline Loci & Query Profile: CNE-2Z & \\
\hline Amelogenin & $\mathrm{x}$ & $\mathrm{x}$ \\
\hline D13S317 & 101213.3 & 101213.3 \\
\hline D16S539 & 91011 & 91011 \\
\hline CSF1PO & 1011 & 1011 \\
\hline TH01 & 79 & 79 \\
\hline vWA & 1416 & 1416 \\
\hline D7S820 & 1012 & 1012 \\
\hline D5S818 & 1112 & 1112 \\
\hline TPOX & 812 & 812 \\
\hline D3S1358 & 1518 & \\
\hline D1S1656 & 1215 & \\
\hline D6S1043 & 1118 & \\
\hline Penta E & 1720 & \\
\hline D18S51 & 13 & \\
\hline D2S1338 & 23 & \\
\hline Penta D & 912 & \\
\hline D8S1179 & 1217 & 1 \\
\hline D12S391 & 2021 & पy \\
\hline \multicolumn{3}{|l|}{ D195433 } \\
\hline FGA & 1821 & $y$ \\
\hline D21S11 & 2730 & \\
\hline \multicolumn{3}{|c|}{$\begin{array}{l}\text { Note: The fop hine Loci ( } 8 \text { core STR lod plus Amelogenin) can be made public to verify } \\
\text { cell identity. In order to protect the identity of the donor, please do not publish the } \\
\text { allele calls from all the STR loci tested. }\end{array}$} \\
\hline
\end{tabular}

Percent Match: $100 \%$

The submitted profile is an exact match for the following human cell line(s) in the reference database (8 core loci plus Amelogenin): CNE-2Z

\section{Explanation of Test Results}

Cell lines with $280 \%$ match are considered to be related; i.e., derived from a common ancestry. Cell lines with between a $55 \%$ to $80 \%$ match require further profiling for authentication of relatedness.

Reference Database Profile was obtained from ExPASy, DSMZ, ATCC or China National Infrastructure of Cell Line Resource STR database. 


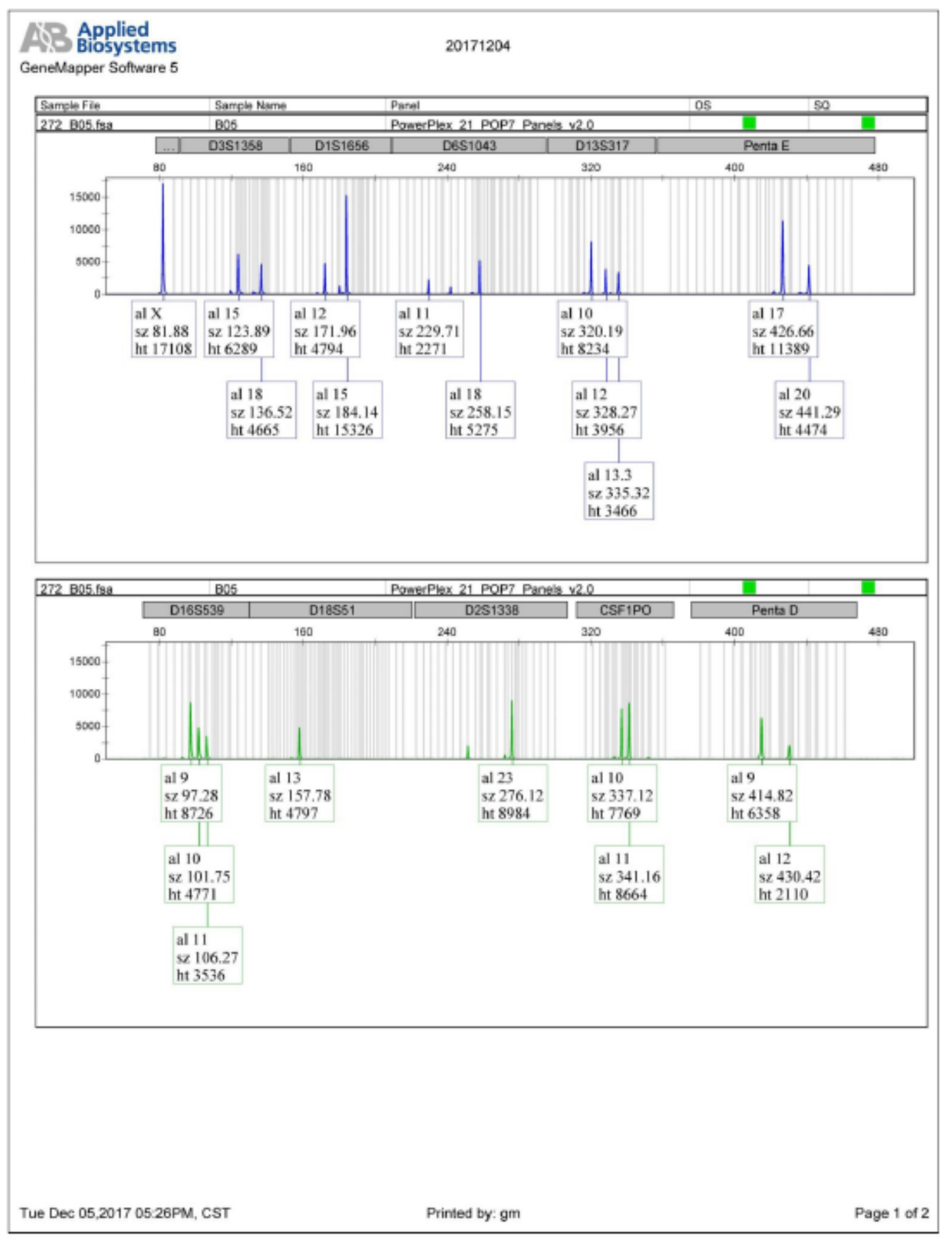




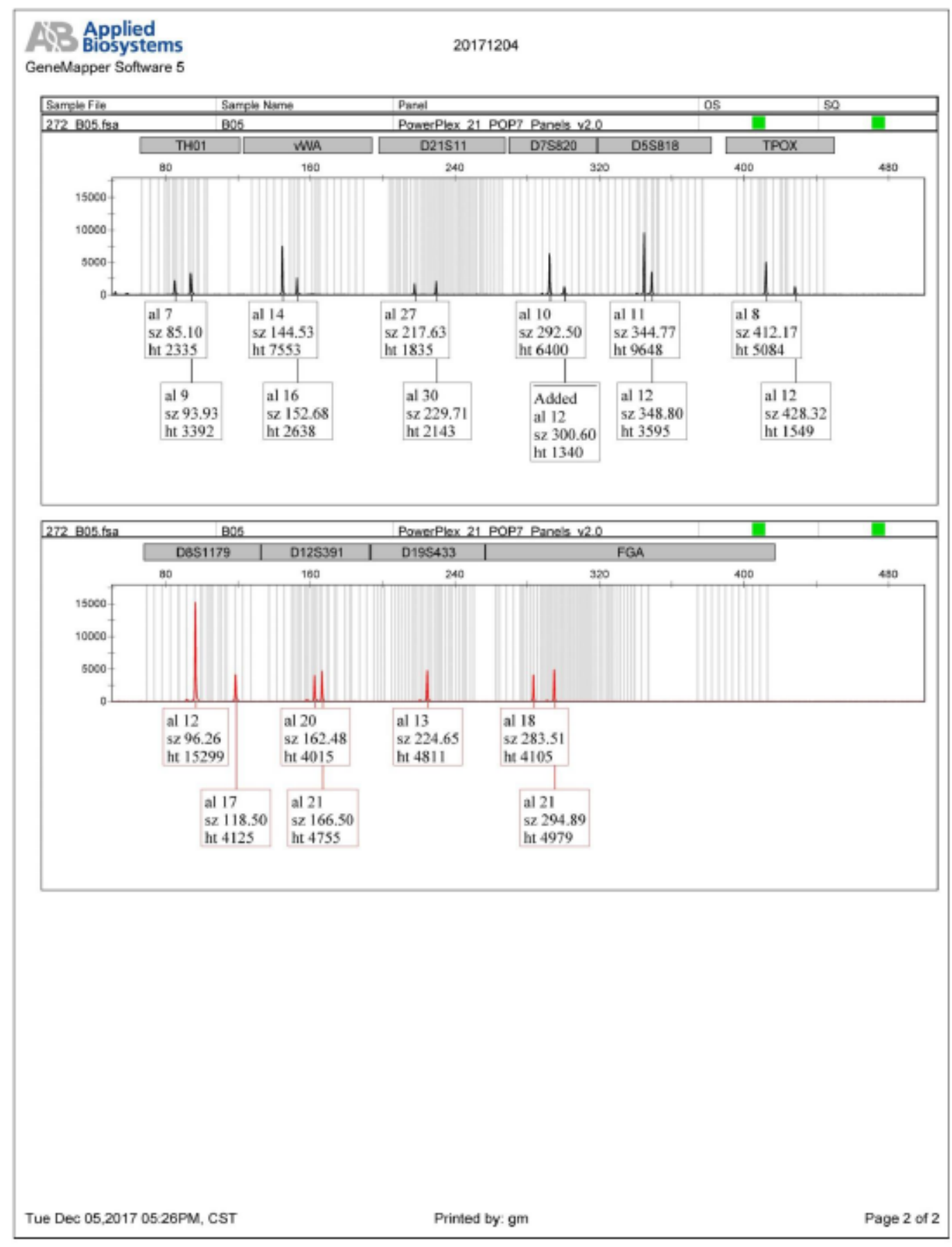

